\title{
Application of dried blood spot for analysis of low molecular weight fraction (metabolome) of blood
}

\author{
Elena E Balashova, Oxana P Trifonova, Dmitry L Maslov and Petr G Lokhov* \\ Institute of Biomedical Chemistry, Pogodinskaya st. 10, 119121, Moscow, Russia
}

\begin{abstract}
The metabolomic blood analysis can be used in clinical laboratory practice to improve the efficiency of diagnostics and risk assessment of diseases, since the current clinical diagnostics is not able to cope with the full range of possible human diseases. According to the latest advances in analytical and computational tools, not more than $1 \mu \mathrm{l}$ of blood is required for metabolic analysis. Therefore, the dried blood spot method is a low invasive method and allows collecting samples with ease of manipulation and minimal costs. In addition, samples can be collected outside the laboratory at home. This review details the dry blood drop method for obtaining a sample for the metabolomic analysis of blood. The review also describes the use of dried blood spot in the workflow with the metabolome blood analysis by direct mass spectrometry, which includes direct injection of extracted from the spot metabolites into the ionization source of quadrupole time-of-flight mass spectrometer
\end{abstract}

\section{The need to study a low-molecular weight fraction of human blood (metabolome)}

A human metabolome is a complete set of low-molecular substances (metabolites), or in other words, a biochemical phenotype of the organism. Metabolome is the result of the interaction of the genotype with the environment. Unlike the genome, transcriptome and proteome, the metabolome is directly related to the biological functions of the body. Metabolites are substrates, intermediates and products of all biochemical reactions occurring in the body, including pathological biochemical processes. Therefore, metabolomic assays can be used in clinical laboratory practice to improve diagnostic efficiency and identify risks of disease occurrence [1-4]. The current clinical diagnosis is not able to cope with the full range of possible human diseases, both existing in the body, and those that can only occur under the influence of a lot of xenobiotics, constantly getting into the body from the air, with food and water, and also which are medicines.

Metabolomic analysis of blood has an un-targeted (review) nature, in contrast to the targeted nature of modern clinical laboratory diagnostics. The ability to simultaneously detect in the blood the entire set of low-molecular substances, including tens of thousands of xenobiotics and markers of various diseases, can significantly improve a person's health and increase the life span. Metabolomic analysis allows regular monitoring of the "health" of a person, controlling the biochemical processes that occur in the body and providing the opportunity to correct them, which is necessary, and perhaps also a sufficient condition for the realization of the longevity laid down in the human genotype [5].

\section{The relevance of the metabolomic blood test in clinical practice}

First, even a person who is considered healthy is always in a state of chronic intoxication. At the same time in the body of each person dozens of toxins are present, causing harm to health and reducing the life span of a person. In this connection, a method is needed that makes it possible to identify xenobiotics (characterized by extreme diversity) in the human body [5].

As already mentioned above, xenobiotics constantly get into the human body from the air, with food and water, as well as being medicines. Food always contains substances that are to some extent toxins for the body and capable of being mutagens or carcinogens, built into metabolic pathways. These can be toxins produced by humans, such as pesticides, industrial waste, by-products of food production, medicines. And can be the products of the life of bacteria and fungi that pollute food and water [6,7]. A lot of toxins arise when cooking food, both at home and in industrial production. For example, proteins of meat and cereal products containing nitrogen, under the influence of high temperatures are converted into acrylamide and benzpyrene, which are the strongest mutagens. When smoking food, $\mathrm{N}$-nitroso substances appear, which become mutagens after they enter the body [8].

Many organic substances, having volatility, easily enter the body during respiration and cause a variety of diseases, including kidneys, the immune system, the hormonal system, blood, and of course, the respiratory system [9]. Any household chemicals, building materials (floor and wall coverings, wood-shaving and varnish-painted materials) also release volatile substances that can enter the human body $[10,11]$. Some of them are found in $98-100 \%$ of the volunteers surveyed $[12,13]$. The literature has even been given the name "sick building syndrome" for characterizing the condition of people who have been staying in a newly renovated or build room for a long time [14]. The carpet is a great source of toxins; more than $90 \%$ of the studied carpets contained a neurotoxin in significant amounts [15]. In another study, in each of the studied carpets, an average of 12 pesticides and their derivatives were found to show that carpet covers are the largest source of such

${ }^{\star}$ Correspondence to: Petr G Lokhov, Institute of Biomedical Chemistry, Pogodinskaya st. 10, 119121, Moscow, Russia, E-mail: lokhovpg@rambler.ru

Received: November 17, 2018; Accepted: November 27, 2018; Published: November 30, 2018 
pesticides as DDT, aldrin, atrazine and carbaryl, which enter our body through the lungs [16].

The toxin database "Toxin and Toxin Target Database" (T3DB) (http://www.t3db.ca) contains more than 40 thousand substances that can enter our body. And these are only those for which the toxic effect is confirmed, and measured over a relatively short period of time. But for most xenobiotics (including drugs), the not time-resolved consequences of their effects on humans are unknown. Any xenobiotic, which has got into the body throughout life, can potentially harm human health and shorten lifespan.

To date, increased attention is being paid to the possible toxic effects of those xenobiotics that are medicines, namely, irrational drug therapy. Irrational drug therapy is observed in $60 \%$ of cases and is the result of mass self-treatment, growing production of generics, simultaneous admission of several drugs by patients, as well as different pharmacokinetics of drugs in different people. The doctor cannot confidently state what medicines are present in the patient's blood at the moment, and at what concentration [17]. Irrational drug therapy leads not only to chronic drug intoxication, thereby reducing life span, but can lead to death. Only in the US, 100,000 people die every year from irrational drug therapy.

Secondly, several thousand human diseases are known. The indication for conducting a diagnosis that can detect them is the manifestation of clinical signs of the disease, that is, when the disease is already causing visible damage to health. Without such manifestations, dozens of diseases persist in the body for a long time and covertly, worsening health and shortening the life span of a person. Therefore, a method is needed to identify the entire variety of possible pathological processes in the human body [5].

Several thousand nosological forms have been described by the World Health Organization (WHO). Unfortunately, in the overwhelming majority, the symptoms of diseases are found even when the disease has developed. Often this development lasts for years, does not manifest itself at all, and the diagnosis is made when treatment can only alleviate the condition of a person, but not heal it. Preventive measures, including laboratory diagnostics, cannot cope with the existing situation precisely because of the excessive variety of the described diseases.

The metabolomic blood test is designed to cope with the two global problems of diagnosis described above - the presence in the body of tens of thousands of xenobiotics, which daily poison the human body and the simultaneous presence of many pathological processes occurring in the same organism. Only a metabolomic blood test, which, as mentioned above, is of an un-targeted nature, can reflect the entire list of low-molecular substances that go beyond the "norm" and "health", that is, xenobiotics and markers of the vast majority of diseases that are dangerous to health.

\section{Mass Spectrometry Analysis of blood plasma metabo- lome}

Most often, metabolomic analysis for the diagnosis of diseases or toxicological research is an analysis of the low molecular weight composition of blood as the main carrier of hormones, substrates and products of biochemical reactions $[18,19]$. A metabolomic blood test can be performed by two methods - NMR spectroscopy and mass spectrometry [2,20-23], but in medical studies for metabolomic analysis, mass spectrometry is mainly used, because of the greater sensitivity of the method. A very detailed description of the mass spectrometry analysis of blood metabolome was described by Lokhov, et al. [24].

The mass spectrum in this case is obtained by direct injection of a blood sample in acidified methanol into an electrospray ionization source of a quadrupole-time-of-flight mass spectrometer. The resulting mass spectrum reflects a metabolomic pattern, the statistical analysis of which in the group with metabolomes of healthy persons allows us to establish the normality of the metabotype, identify xenobiotics, low molecular weight biomarkers of diseases, and apply metabolomic diagnostic signatures [24].

It should be noted that for metabolomic analysis, not more than 1 $\mu \mathrm{l}$ of blood is needed, therefore the volume is determined exclusively by the convenience of manipulation and can be carried out even outside the laboratory. Given the focus of this review, it is necessary to consider in detail the use of the "dried blood spot" (DBS) method to obtain a sample for metabolomic analysis.

\section{Historical background on the use of DBS in biomedical research and medicine}

The idea of using blood collected on a paper card from cellulose is attributed to Ivar Christian Bengu, the father of modern clinical microanalysis [25,26], who in 1913 determined glucose in eluate of DBS [27]. Subsequently, several researchers used the DBS in serological testing to diagnose syphilis [26]. In 1924, Chapman summarized the benefits of DBS analysis, emphasizing four points that are still relevant today: (1) compared to conventional venipuncture, requires a much smaller amount of blood, and this fact was the most important in pediatric diagnostics; (2) collection of blood is simple, non-invasive and inexpensive; (3) the risk of bacterial contamination or hemolysis is minimal; and (4) DBSs can persist for a long time, practically without worsening the results of the subsequent analysis $[26,28]$. In addition to the use of DBS in syphilis testing, as early as 1953, the method was used for the detection of antibodies against measles, mumps, poliovirus, parainfluenza virus and respiratory syncytial virus (RSV) [26], as well as for detection antibodies to schistosome in the DBS taken from endemic areas and analyzed more than three months later [29].

In 1963, fifty years after the first report of Bang [26,30], Guthrie published his famous method for the diagnosis of phenylketonuria in the DBS derived from the newborn's heel $[31,32]$. Although no this analysis is currently being conducted, it still forms the basis of the modern screening programs for newborns around the world [33]. The initial semi-quantitative test for phenylketonuria screening, developed by Guthrie, based on the inhibition of the bacterial culture, was very sensitive, but had poor efficacy [34]. Thanks to the introduction of advanced analytical methods that expanded the testing capabilities and improved its effectiveness, the possibilities of using newborn screening with the DBS were expanded. In many medical centers, the testing began to include screening for congenital hypothyroidism and cystic fibrosis [35].

Although the DBS has since been regarded as a generally accepted method for collecting, storing, transporting and analyzing human blood [29], its use in laboratory practice for decades has remained focused mainly on the diagnostics of infections and the screening of newborns for hereditary metabolic disorders [36,37]. Since 2005, however, new and innovative techniques have started to appear using DBSs. This led to an almost exponential increase in the number of relevant scientific publications on the DBS from 50 to almost 450 annually at the present time. Among the new areas of application are such diverse fields as 
toxic and pharmacokinetic studies, metabolic profiling, therapeutic drug monitoring, forensic toxicology, environmental pollution control [37,38].

\section{Use of DBS in medical diagnostics}

Advantages of using DBSs before liquid blood samples (whole blood, plasma, serum) are obvious and are as follows: 1) simplicity and accessibility of reception among any population groups; 2 ) blood sampling does not require the conditions of a clinical laboratory; 3 ) the samples do not require urgent additional processing (for example, the production of serum); 4) small volume gives compactness during storage and transportation; 5) samples are less dangerous to handle; 6) low probability of contamination of samples.

However, a small sample volume also means that the concentration of the target analyte is potentially rather low (it may be less than 1 $\mathrm{ng} / \mathrm{L}$ ), which requires an extremely sensitive method for detecting and quantifying the substance. Currently, the most common method for the analysis of DBSs is mass spectrometry [39-42]. The use of mass spectrometry (MS) in clinical laboratories led to a more frequent use of the DBS method, not only for screening newborns, where MS began to be used in the 1990s [43].

Researchers began to find a new application of DBS for mass spectrometric analysis. The DBS method has been used for a number of clinical purposes, including drug toxicology and sports doping screening. But scientists and laboratory workers, on the other hand, have problems with how to ensure optimum sensitivity, reproducibility and accuracy in mass-spectrometric analysis of DBSs.

The first application of mass spectrometric analysis to dried blood spot was reported more than 40 years ago (in 1976) for the detection of fatty acids by chemical ionization [44]. By the mid-1980s, when gas chromatography (GC) became the main method for separation and analysis of small volatile molecules, the derivatized fatty acids were measured in DBS samples using gas chromatography coupled with mass spectrometric detection of substances (GC-MS) [45]. In the 1990s, when electrospray ionization became commercially available, liquid chromatography associated with mass spectrometric detection of substances (LC-MS) was introduced into analytical tools for neonatal screening laboratories, which led to an expansion of screening to phenylalanine and tyrosine, two early markers $[43,46]$. To date, dried blood spot mass spectrometry (tandem mass spectrometry) is a workhorse for many laboratories for screening newborns around the world; and additional tests are added to the list. In addition to screening for newborns, tandem dried blood mass spectrometry (DBS-MS) was used for epidemiological screening, analyzing blood spots for the presence of benzoylecgonine (the primary cocaine metabolite) [47].

At present, the list of substances and biomarkers detected in the DBS by mass spectrometry is growing every day [48,49], having long gone beyond the screening of newborns. It is noteworthy that the DBS analysis with the MS now covers translational studies and clinical diagnostic tests in the field of therapeutic drug monitoring (TDM); pharmacokinetics; toxicokinetics; forensic medical examination; endocrinology and metabolism; and in other areas of bioassay. Therapeutic and toxicological analyzes of various drugs are most widely reported in the literature using DBS-MS (both LC-MS and GC-MS) $[50,51]$. Also, DBS-MS is now often used in sports doping tests to detect anabolic, ergogenic and masking agents [52-54].

Regardless of clinical application, there are some analytical features. A number of parameters can affect the accuracy of measurements in the DBS [44]. Proceeding from this, a general step-by-step protocol is needed for use in the laboratory of the DBS for the analysis of low molecular weight fraction of blood [55-57]. The researchers proposed the following procedure for the preparation and treatment of DBSs: (1) blood sampling; (2) receiving blood spots; (3) drying blood spots; (4) storage and transportation; (5) elution of the DBS; and, finally, (6) mass spectroscopic analysis of the DBS eluates.

It should be noted that this order requires adaptation to the tasks posed by direct mass spectrometry used for metabolic blood analysis. That is, when a sample of blood in acidified methanol comes through direct injection into an electrospray ionization source of a quadrupoletime-of-flight mass spectrometer.

\section{Preparation of a DBS sample}

The DBS preparation includes the following processes: 1) blood collection from the patient; 2) obtaining blood spots on the carrier; 3) drying; and 4) transportation and storage of the DBS sample. In addition to the usual requirements for routine blood collection, additional problems arise in DBSs preparation, including the quality of the DBS sample (which depends on the collection of the sample and the differences in the spots obtained), the choice of the carrier for collection, the method of transport and storage. Moreover, biological factors such as sample viscosity, hematocrit level, and the nature of the analyte (for the targeted assay) can lead to a change in the quality of the sample.

To control the quality of this stage, the following guidelines have been issued: on patient identification - "Safety and Quality Improvement Guide Patient Identification and Procedure Matching. Australian Commission on Safety and Quality in Health Care" [58]; on sampling of capillary blood - "Guidelines on Drawing Blood: Best Practices in Phlebotomy, Capillary blood sampling. World Health Organization" [59]; for the selection of filter paper and for applying a sample to the filter paper - "Blood Collection on Filter Paper for Newborn Screening Programs; Approved Standard" [60]; and on packaging and transportation of a sample of the DBS - "Laboratory Quality Assurance and Standardization Programs. Guidelines for the Shipment of Dried Blood Spot Specimens. USA: Centers for Disease Control and Prevention" [61].

\section{Blood sampling}

The technique of blood sampling is important for an accurate analysis of the DBS. Capillary blood sampling is a common approach, as it usually requires less sample volume and more friendly to the patient, compared to venipuncture. As the World Health Organization (WHO) and the United Nations International Children's Emergency Fund (UNICEF) certify the quality of the DBS samples and ensure the safety of health care workers by providing guidelines and instructions for the DBS samples [62,63]. Because of the high occurrence of artifacts probability, special conditions of capillary blood sampling for the DBS are allocated, including: selection point injection, pricking device size, the injection depth, the flow rate during capillary blood, droplet size, uniformity of diffusion of the sample on a carrier, contamination of the DBS through prolonged exposure to air or light during the drying process. It is important to note that WHO indicates that "work with the DBS requires the same precautions with regard to biological hazards as whole blood or plasma [63].

When taking blood samples for metabolic analysis, it is necessary to take into account certain features of metabolites. Lokhov, et al. indicate the following: firstly, this is the strong variability of their concentrations in the blood plasma. Thus, the distribution of metabolites in the body 
varies depending on its state and time of day, which imposes stringent requirements on the conditions for obtaining samples for analysis, i.e. blood samples should be taken at the same time. The second feature - this is the lack of species-specificity of the majority of metabolites, which leads to a significant influence, for example, food substances on the assay results. Therefore, blood sampling should be carried out before meals [5].

\section{Selecting the carriers for the DBS}

From the literature data it is known that the collection of DBS samples is performed on one of two types of untreated solid substrate: pure cotton filter paper and glass microfiber (Figure 1). The choice of carrier, including thickness and density, affects the rate of adsorption and dispersion of capillary blood. It has been shown that, for example, the rate of membrane nonspecific adsorption of the analyte decreases with the use of glass microfiber paper [64]. Accordingly, differences in the use of these two solid carriers can cause changes in the characteristics of the DBS samples, leading to potential differences in blood volume in the spot, the stability of the analyte, the number of target analytes, and the possibilities for further analysis [65]. These specific pre-analytic differences in DBSs require standardization.

A
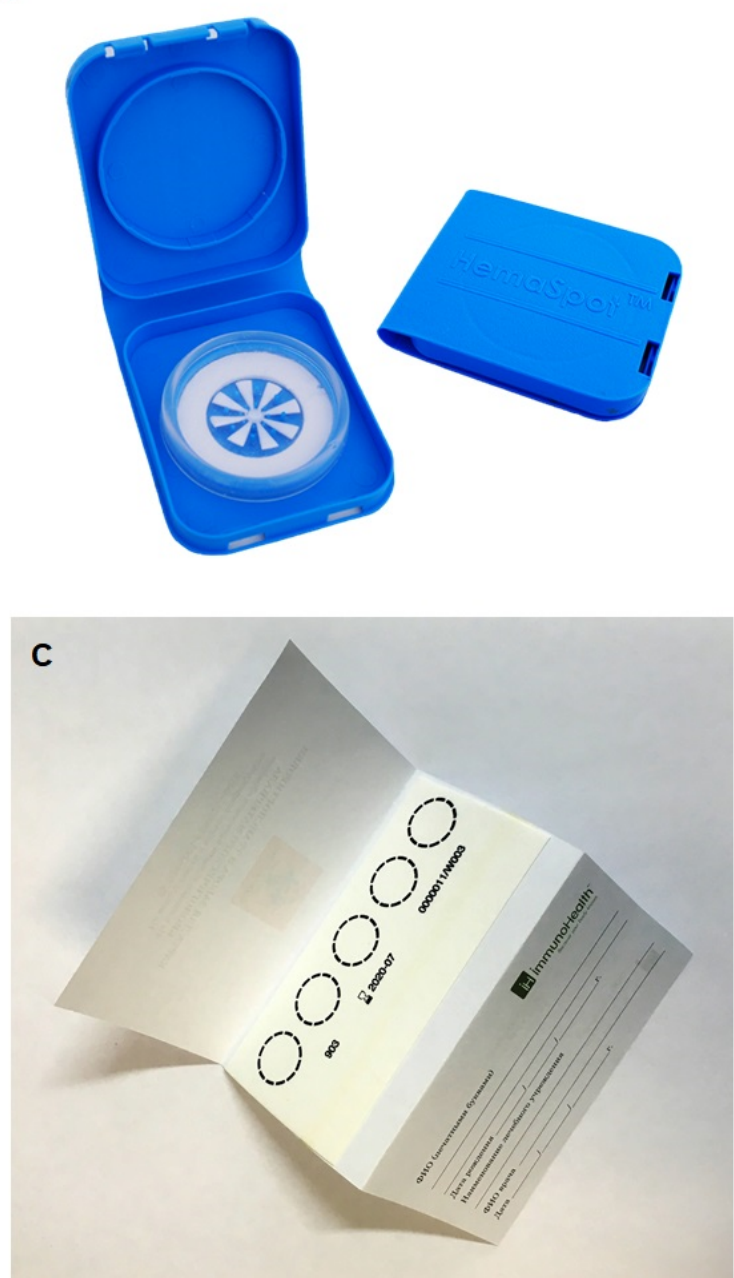

Clinical and Laboratory Standards Institute (CLSI) offers a guide NBS01-6 for DBS obtaining [60]. The CLSI guidelines recommend the use of one of two special cards: Whatman 903 and Ahlstrom 226. Both filter papers are approved by the Food and Drug Administration (FDA), the Neonatal Screening Quality Assurance Program (NSQAP), and the Centers for Disease Control and Prevention (CDC) [60]. This guide is the starting point for selecting a carrier for obtaining a DBS.

\section{Applying the sample to the filter paper}

Both the Whatman 903 and Ahlstrom 226 cards have a printed collection area to provide a sample volume within 50-75 $\mu \mathrm{l}$ [60]. A drop of intact capillary blood should be applied to the middle of the collection area to provide radial dispersion to the isolated spot boundary. This lateral distribution is uneven, as shown by autoradiography [66]. Usually, the concentration of biomarkers decreases along the edge of the DBS, while the middle is subject to the "volcanic effect" (paper chromatography effect) and sometimes has a patchy pattern (thicker patterns) [67]. The physical characteristics of the DBS sample can also be affected by the hemoglobin and hematocrit levels of the patient; which also affects the area of spread of the drop of blood.
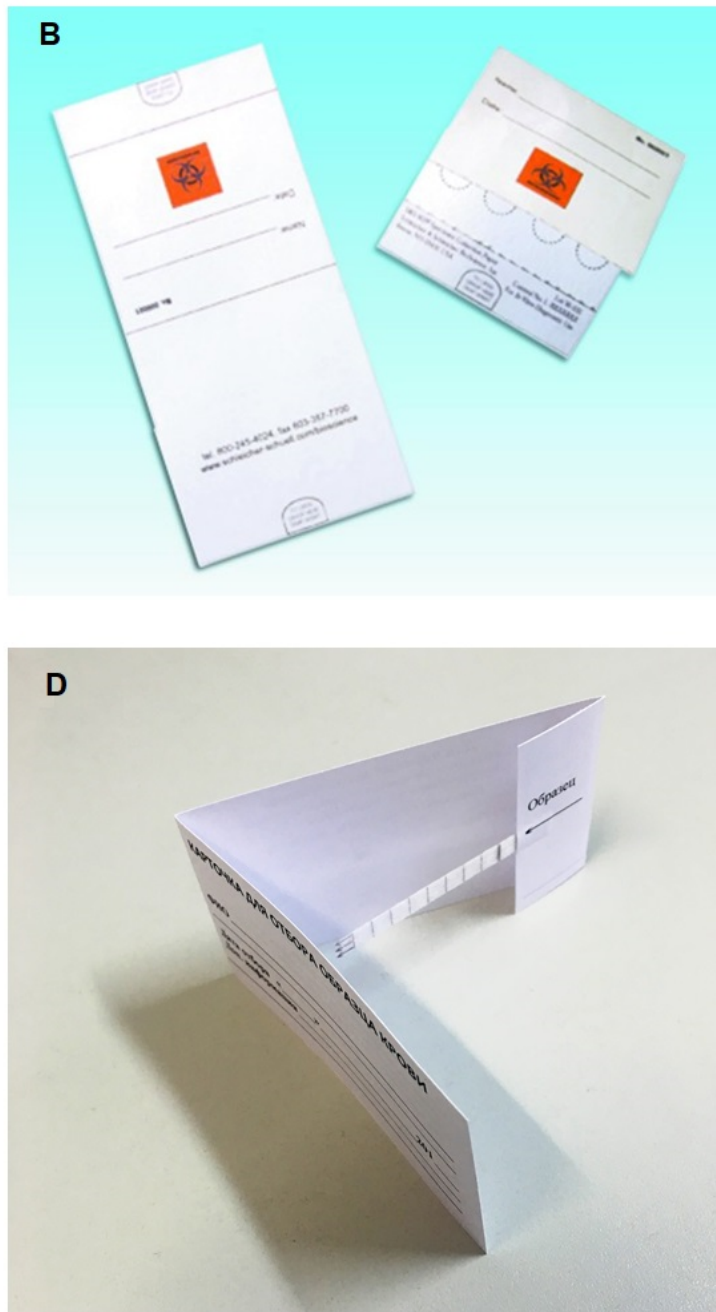

Figure 1. Adsorption materials for sample collection using the dried blood spot method. A, cartridge containing an absorbent paper HemaSpot ${ }^{\mathrm{TM}}$-HF (Spot On Science). B, Whatman's protein saver card Whatman ${ }^{\text {TM }} 903$ Protein Saver Snap Apart Cart (7078417 GE Healthcare Life Science). C, card ImmunoHealth ${ }^{\text {TM }}$ Whatman 903 (analogue Whatman ${ }^{\text {TM }} 903$ Protein Saver Snap Apart Cart). D, glass microfiber strip ImmunoHealth ${ }^{\mathrm{TM}}$. 
Variations in the hematocrit will affect the relative percentage of plasma in the spot. This is important for analytes, mainly found in serum/plasma, because the relative amount of plasma in discs cut from different spots can vary, especially when the hematocrit is extremely high or low [68]. Samples of whole blood with a higher hematocrit tend to be less distributed on the filter paper (i.e., to form a smaller blood-spot diameter), and therefore the diffusion distance of the target analyte is shorter in such samples. The reverse effect is observed for blood samples with low hematocrit. As a result, the concentration of the analyte (as compared to the sample with "normal" hematocrit) can be determined higher or lower due to a change in the analyte distribution $[69,70]$. Therefore, for an accurate quantitative determination, ideally, it is recommended to determine the patient's hematocrit either in a separate sample of capillary blood collected at the time of DBS production, or directly from the DBS-card.

\section{Preservation of analyte stability (drying, storage and transport conditions)}

Samples DBSs must be completely dried before transport and/or storage. It was shown that rapid drying and storage in low humidity conditions improves the stability of DBS samples [51]. The length of time required for drying in air will depend on local environmental conditions, such as air conditioning, room temperature and humidity. DBS drying usually takes from 90 minutes to about 4 hours, and ideally, the DBS sample should not be exposed to direct sunlight during this period.

A properly dried sample can then be placed in an envelope or a suitable container for logistically simple and economical transport [51]. Since the low volume of the dried sample significantly reduces the risk of transmission of infection compared to other biological samples $[71,72]$, it can be transported in small light packages that do not require a special temperature. This is a significant advantage over the often cumbersome and expensive processes associated with the transport of liquid biological samples [51].

Once the DBS samples are received at the destination, the size and properties of the DBS samples make their storage relatively easy, because, first, they require a minimum space, and secondly, they do not require a special temperature, they can be stored at room temperature.

However, one should keep in mind that the stability of various analytes in the DBS can be quite variable. Thus, it has been shown that the stability of numerous blood biomarkers in the production of DBS on paper at room temperature has been confirmed for at least 7 days [64]; stability of pharmaceuticals, opiates and nucleic acids - for several months [73,74], vitamin D - for more than 20 years [75]. It was also shown that freezing at $-20^{\circ} \mathrm{C}$ or $-80{ }^{\circ} \mathrm{C}$ enhances the stability of DBS samples; often increasing the stability of analytes from several days/months to several years [76-78]. In addition, according to some literature data, some metabolites have greater stability in the form of dried paper drops of whole blood, compared to plasma [79].

As already mentioned, in addition to the storage time, many factors can affect the stability of the analytes: the type of filter paper, direct sunlight, temperature, humidity, and the nature of the target analyte. In the literature, it is possible to find the methodological results of some laboratories used in the preparation of the DBS to improve stability, for example: 1) a rapid decrease in the $\mathrm{pH}$ of the blood sample to increase the stability time of some structurally unstable drugs that degrade during the drying process; 2) the use of ethylenediaminetetraacetic acid (EDTA) as an anticoagulant for the preservation and stabilization of DBS samples for the determination of enzyme activity [80,81]. Obviously, if there are so many variations in the stability of the analytes, it is necessary to develop special protocols for obtaining and storing DBS samples. In the literature, there are many recommendations adapted to specific tasks and objectives. In a review by Zakaria R. et al. there is a summary table that includes the stability time of the DBS samples for various analytes according to the literature [49].

\section{Analysis of DBS samples}

The solid form of the DBS sample is incompatible with most analytical methods and requires the elution of a sample from a paper carrier. Accordingly, the sample analysis process typically includes three main steps: 1) primary sample preparation, 2) pre-treatment and 3) sample analysis. The analysis can be further divided into chromatographic separation and mass spectrometric detection. But in accordance with the metabolomic research, the quality of the entire analytical process must correspond to the fact that the analysis of samples is carried out by direct mass spectrometry.

\section{Primary preparation of samples}

Cutting a disc with a sample: Sample preparation usually begins with cutting out a portion of the dried blood spot from a paper carrier manually or in an automated manner. As a rule, in order to minimize sample differences due to which part of the spot is cut out, it is recommended to cut the disc all the time either from the center of the dried blood spot or closer to the outer edge [82]. The size of the cut out disc can vary from $3-6 \mathrm{~mm}$ to the whole spot, depending on the method.

In the literature, methods have been proposed to minimize the problems of hematocrit variations and the associated difficulties in the preparation of samples. Strategies to overcome the effect of hematocrit include:

\section{Preliminary cutting of disks from the paper carrier;}

2. Apply a small volume of whole blood (e.g., $10 \mu \mathrm{l}$ ) to a pre-cut small disc ( 3 or $6 \mathrm{~mm}$ ) and analyze the entire disc [83-86];

3. Use of a two-layer polymer membrane to obtain a dried plasma spot from a whole blood sample to be analyzed after solid-phase extraction [87];

4. Development of a new DBS sample collection card to obtain a plasma sample of a specific volume $(2.5$ or $5.0 \mu \mathrm{l})$ from a whole blood sample. The prospective advantages of this matrix include improved reproducibility and selectivity of the assay, together with a simplified procedure for extracting the sample and eliminating the hematocrit effect [88].

Elution: To analyze the analyte, it is first required to isolate it from a paper carrier using a suitable extraction buffer. Effective elution of analytes from the DBS is a complex process, and there is always the possibility of loss of the analyte due to inefficient extraction. A poor elution of the sample occurs due to incomplete extraction or degradation of the analyte. Therefore, the selection of the optimum extraction buffers may be different from the compound to the compound. As an example: pure methanol is considered a common solvent for low-extraction substances from the DBS sample [89]. Water, on the other hand, degrades the interaction between the cellulose and the hydroxyl groups of the target analyte and the partial addition of water preceded by organic extraction increases its effectiveness in some cases (e.g., for antiviral drugs) [90]. To achieve extraction of the 
analyte with maximum extraction efficiency, it is necessary to optimize the extraction parameters, including the composition of the extraction solution, the duration, the temperature and the application of additional ultrasound treatment, for each individual target metabolite $[68,91]$.

\section{Preliminary treatment of the sample.}

Various approaches to sample preparation have been proposed, depending on the molecular characteristics of the target compound. Methods of preliminary processing of samples, either in combination with each other or separately, represent a classical process of sample preparation: 1) protein precipitation; 2) liquid-liquid extraction; 3) solid-phase extraction; 4) liquid extraction on the substrate; and/or 5) derivatization.

The extraction and derivatization procedures are largely time consuming and labor intensive. While derivatization is not required for most plasma analytes analyzed with LC-MS/MS, in many cases, DBS analysis needs to improve sensitivity by compensating for a small sample volume. However, since the derivatization process extends the total analysis time, it is considered to be a limiting factor and is the driver for the development of extraction methods that facilitate preanalytical processing of DBS samples.

Automatic sample preparation was introduced, directly related to the LC-MS/MS system, which reduces the time and cost of the process. Protein precipitation is a simple and popular method of automation that is used for TDM [92]. However, after one precipitation procedure, salts and other endogenous analytes are still present which can cause ion suppression in the MS process. Solid-phase extraction conjugated to LC-MS/MS is designed to automatically facilitate desorption of samples and is effective both in time and in cost by a method for analyzing DBSs [74,93-96]. Compared with protein precipitation, solid phase extraction is an improved purification of the sample [97]. There are specific problems with the use of automated extraction in comparison with the methods of autonomous extraction, which can also be a significant source of analysis errors: an inhomogeneous mixture of the internal standard with the analyte in the extract; dilution of the sample, and as a result, broadening the band in chromatographic separation and/or difficulty separating the extract on the analytical column [44]. Accordingly, in the process of developing a method to address these problems, certain strategies are required.

A technology has been developed that allows direct analysis of the DBS without the need to transfer to a liquid phase or elution. As described by the manufacturer, "Liquid Microjunction Surface Sampling Probes (LMJ-SSP)" are self-priming devices in which liquid is sucked off from an interesting surface and pumped to a mass spectrometer for complex extraction and ionization (Prosolia, 2014). Using LMJSSP technology, the analyte of interest can be directly extracted from different surfaces and detected by a mass spectrometer in a short period of time with minimal sample processing [98]. The LMJ-SSP device associated with MS was used to detect proteins in a DBS sample [99], for detecting hemoglobin by direct tandem mass spectrometry [100], and for detecting therapeutic drugs [101,102]. The use of this method for the detection of thiophan preparation has shown that such direct derivatization on the spot is also effective both in time and in cost, as an alternative to the sample preparation procedure [103,104].

\section{Analysis of samples}

Gas chromatography is known as a cheaper and faster separation method compared to LC, since longer and more compact capillary columns are used that improve resolution. Analytical methods of GC-
MS often offer higher separation ability and effective reproducibility in comparison with LC-MS. GC-MS is suitable for the analysis of volatile and semi-volatile organic substances, it can be used both for identification of unknown substances in mixtures and for quantitative analysis of target substances. These methods examine steroids, metabolites, and therapeutic agents using DBS samples [52,103,105107]. However, the use of GC is limited to small molecules that are soluble in a certain solvent, volatile and heat-resistant, since derivatization is frequently required to convert nonvolatile molecules to volatile ones $[108,109]$.

LC is the preferred method for analyzing temperature-sensitive analytes without limiting the molecular size. In addition, unlike GC, target compounds interact with both the mobile phase and the solid phase, leading to better selectivity [109]. The choice between GC or LC depends on the required sensitivity and characteristics of the target analyte. Although GC-MS provides selectivity, sensitivity and reliability for many DBSs analyzed, literature shows that it is not as popular as LC-MS/MS [49]. This is probably due to the improved specificity and sensitivity provided by LC-MS/MS for DBS analysis due to a much faster and usually more economical process compared to GC-MS $[110,111]$. In addition, the improved HPLC technology allowed to increase resolution of peak separation (even more efficient than GC) $[108,109]$.

With the introduction into the analysis of two-dimensional chromatography (2D chromatography) (applicable to both GC and LC), separation efficiency, analytical sensitivity, thoroughness and accuracy of quantitative analysis were improved. The $2 \mathrm{D}$ chromatography process reduced matrix and transport effects for DBSs, and related inaccuracies were reduced [112,113]. With the further addition of automated extraction coupled to the $2 \mathrm{D}-\mathrm{C}$ system, sensitivity and specificity become maximum when combined with a triple quadrupole tandem mass spectrometer or high-resolution quadrupole mass spectrometer (QTOF) [97,114].

Advances in ion source technology have increased the sensitivity of the assay, both for polar and nonpolar analytes from DBS samples [115117]. Modes of selective or multiple monitoring in MS/MS detection significantly improved the specificity of the analysis, improving the linearity and detection limits [44,118].

Release from the phase of pre-analytical purification and chromatographic separation, DBS samples [119] use direct MS methods and LMJ-SSP methods in combination with MS. Desorption electrospray ionization (DESI), direct analysis in real time (DART), and direct electrospray ionization mass spectrometry (ESI-MS) are used to generate ions from the surface, thus avoiding purification or derivatization processes [119-126]. However, it should be kept in mind that eliminating primary purification and sample separation can lead to loss of sensitivity and accuracy due to destroyed interferences of metabolites [125]. Thus, the use of direct MS methods is recommended to increase the sensitivity and accuracy of the measurement $[111,127]$. Ultimately, the time efficiency and throughput must be balanced with the achievement of the desired results.

\section{Mass Spectrometric Analysis of Blood Metabolome}

The non-target (overview) nature of metabolic analysis is provided by the use of mass spectrometers with a high resolution of mass detection. At the same time, the mass spectrum is a "window" with a width of $1000 \mathrm{Da}$, in which all low-molecular substances are reflected with a resolution of $0.0001 \mathrm{Da}$ in a range of concentrations up to $10^{5}$, 
capable of ionizing in the electrospray ionization source of the mass spectrometer. Given that only a small amount of low molecular weight substances is not completely ionized into electrospray, metabolic analysis is potentially suitable for detecting all abnormalities in the low molecular weight composition of blood [5].

For medical purposes, metabolomic blood analysis is carried out by direct mass spectrometry, that is, by direct injection of the analyzed sample into the ionization source of the mass spectrometer, without prior isolation, separation or enrichment of the analytes under study (Figure 2). Since any additional stages of sample processing can introduce distortions into the overall picture of the metabolome, the "release" from them allows the analysis results to be brought closer to the "real" metabolome as much as possible. The use of modern mass spectrometers with a high resolution of the detection of masses of substances allows obtaining the "real" metabolome. For example, the use of a hybrid quadrupole-time-of-flight mass spectrometer (Q-TOF) for direct mass spectrometry makes it possible to detect 15,000 ion metabolites in the blood in only one mode of mass detection. This number of detectable ions should be sufficient to reflect the entire low molecular weight composition of human blood [128].

Lokhov and co-authors [5] distinguished the following main characteristics of the metabolomic blood test on the basis of direct mass spectrometry.

1) The method analyzes low molecular substances up to $1000 \mathrm{Da}$, which include toxins, drugs and metabolites of both physiological and pathological biochemical reactions taking place in the body.
2) The method does not analyze metal ions.

3) The analysis is not targeted, which makes it possible to identify all the variety of non-normative substances in the blood.

4) It is possible to identify not every non-normative substance found in the blood.

5) The mass spectrometric analysis of the blood sample takes place "as it is", that is, without preliminary sample preparation and with the direct injection of the analyzed sample into the electrospray ionization source of the mass spectrometer.

6) It is possible to use any biomaterial: blood plasma, venous blood, capillary blood, a drop of blood dried on a filter paper.

7) For the analysis, it is necessary to have a micro-quantity of biomaterial (not more than $1 \mu \mathrm{l}$ ).

8) The effectiveness of metabolomic analysis depends on the power of the mass spectrometer used; for effective analysis, the resolving power of a mass spectrometer must be not less than 20,000 (the ratio of the height of the mass spectrometric peak to its width, measured at the middle of its height); accuracy of measuring the mass of 1-2 ppm and the width of the dynamic range of measurement of mass is not less than $10^{5}$.

9) Use a mass spectrometer with an electrospray ionization source (soft ionization almost does not destroy and well ionizes low-molecular substances);
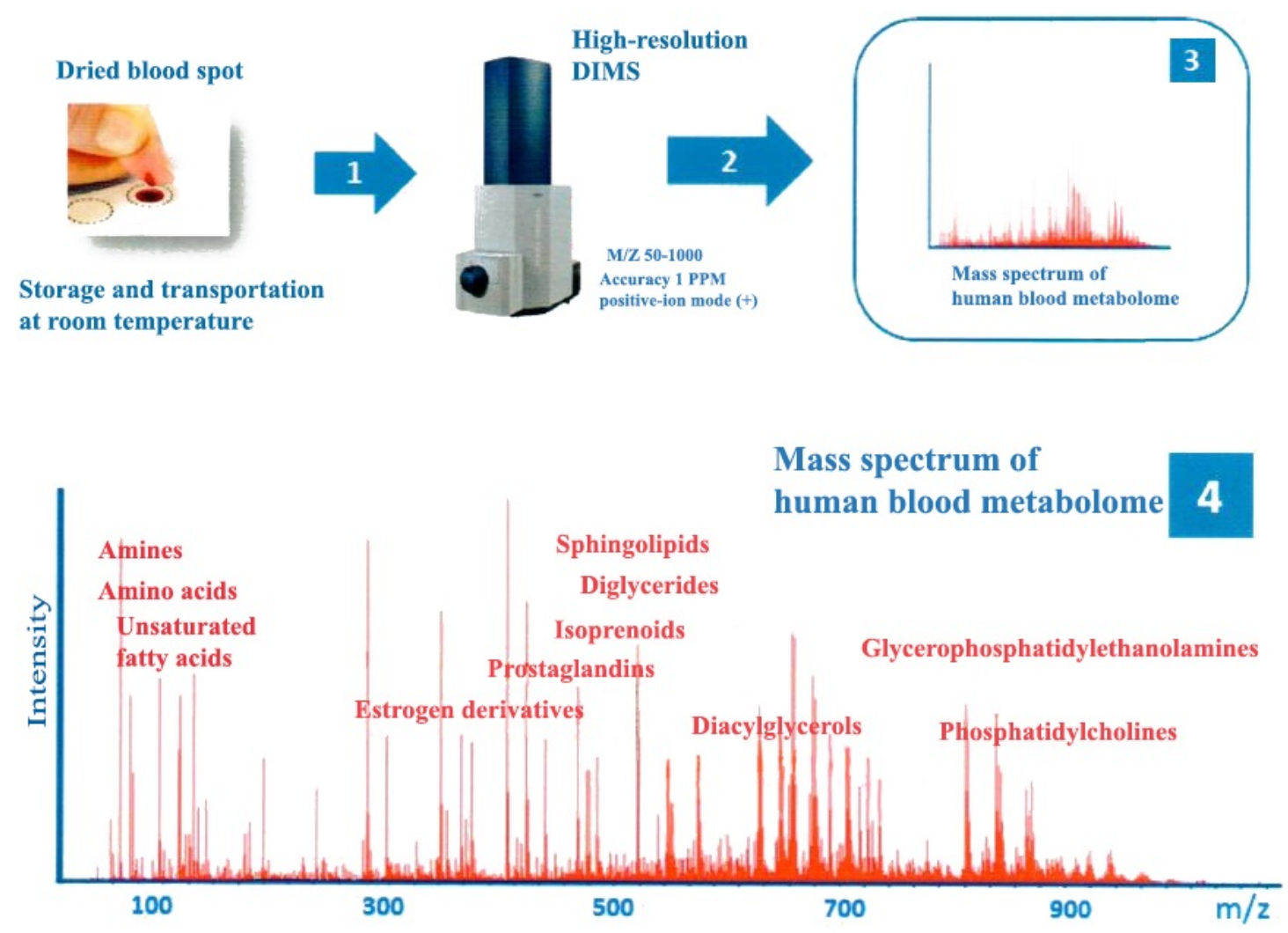

Figure 2. The scheme for obtaining a human blood metabolome based on the MS data. A dry spot of capillary blood on the filter paper is sent to the laboratory for metabolomic study. The low molecular weight substances are extracted from the blood sample (1) and using Direct Mass Spectrometry (DIMS) (2), the mass spectrum of blood metabolites is obtained (3). The example below represents the mass spectrum (4) obtained by direct infusion of blood metabolites into the Electrospray Ionization Source of a quadrupole-time-of-flight mass spectrometer (Micro TOF-Q, Bruker Daltonics) set to detect positively charged ions. The mass-to-charge ratio is 50-1000. The mass spectrum shows the main registered groups of metabolites 
10) The mass spectrometer is used in the mode of positively charged ions detection (the low-molecular composition of the blood is most fully reflected); measurement in the negatively charged ions detection is an additional option.

11) Alignment of mass-spectrometric data is an obligatory step before statistical processing of mass-spectrometric data.

12) The detection of non-normative metabolites is performed by statistical processing of data, by calculating the probability that the concentration of the substance in the blood is not normative.

13) Identification of non-normative substances is carried out by standardized methods and includes 4 levels of authenticity of identification.

14) The correspondence of the metabotype to the normal one, as well as the degree of deviation of the metabotype from the normal one, is estimated by statistical calculation of the probability that the metabotype belongs to a sampling of the healthy individuals.

15) The results of metabolic analysis do not have the status result of clinical laboratory diagnosis or a chemical-toxicology study.

16) The interpretation of the results of the analysis is performed by a physician-biochemist or a toxicologist.

\section{Quality control}

In conclusion, important points concerning the validation criteria and the introduction of the method for the MS analysis of DBS in clinical laboratory practice should also be discussed. The European Bioanalysis Forum (EBF) described the details of the DBS sample analysis methodology to provide specific recommendations for the validation of DBS methods [129]. The document containing EBF recommendations includes specific points: variability of cards for the collection of DBSs; sample variability; uniformity of the DBS; the effect of localization on the spot of the place from which the disk is cut out; stability of the sample; influence of physical parameters of blood; influence of the carrier; extraction; the application of the internal standard (IS) and internal quality control (IQC). In addition to the details presented in this document, it is necessary to consider the suitability of the method for use in clinical practice.

\section{Card for collecting DBSs}

To avoid problems with the variability between cards, the calibrator and control material must be prepared using the same type/ manufacturer of the DBS collection card as for patient samples. If several types/card manufacturers are used, a comparison method is required to determine comparative card stability, extraction, and media effects [130].

\section{Hematocrit effect}

As mentioned earlier, the physical behavior of the whole blood drop applied is influenced by various parameters, such as; the level of hematocrit, the degree of hemolysis and the type of anticoagulant (if it was used). Currently, hematocrit is recognized as the most significant parameter affecting the characteristics of the blood spot (drying time, diffusion and homogeneity) and the reproducibility of the analysis. The effect of the hematocrit is more significant when not analyzing the entire sample of the DBS, but a disc cut out from the spot. Therefore, the development of a validation method for the application of DBS samples should also include studies of the effect of hematocrit change on measurement and analysis results [131].

\section{Application of internal standard}

Including an internal standard (ISTD) in the processing of DBS samples is an important step and should ideally take place at an early stage of the process. 1) Collection cards pre-treated with ISTD may be prepared prior to application of blood. This ensures that both the ISTD and the analytes are subjected to the same carrier and extraction effects. However, this approach can not logically be feasible in multiple studies. 2) Typically, manual extraction methods use the ISTD integration approach in the DBS at the stage of solution preparation for elution/ extraction. In this case, ISTD is extracted together with the target analyte. 3) Adding ISTD to the sample at the extraction/preparation stage is another simple alternative. However, since ISTD does not fully interact with the carrier, the variations in elution are not taken into account. 4) When using the DBS sample preparation technology in an automated mode, ISTD is sprayed onto a blood stain prior to extraction, using Touch-spray technology [132-134].

\section{Sample contamination}

The contamination of the sample is an important problem for the analysis of DBS-MS. Pollution can have different sources, including: physical contact "card-card" during storage; contact "spot-stain" through the device for cutting discs or during subsequent preparation of the sample through a pipette-dispenser or analytical column) [129]. To avoid this problem, either a purification step or an empty map between the samples is recommended [135].

\section{Internal quality control}

The preparation of samples for internal quality control (IQC) for DBS analysis requires special recommendations, as compared to liquid phase biological samples. The main problem with internal quality control is that the carrier conforms to the carrier on which a patient's blood drop is applied [130]. Ideally fresh samples of whole blood, without hemolysis signs, with a closely matched hematocrit level in the study group, should be selected for the preparation of IQC samples [136]. The samples should be applied and eluted with patient samples.

\section{External quality control}

External quality assessment (EQA) is considered important tools for assessing the reliability of an analytical procedure, as well as for monitoring the quality of laboratory indicators. The UK National External Quality Assessment Service (UK-NEQAS), the European Research Network for Evaluation and Improvement of Screening, Diagnosis and Treatment of Hereditary Metabolic Disorders (ERNDIM) and the US Centers for Disease Control and Prevention (NSQAP) provide various schemes for DBS testing for screening purposes newborns. However, there is no additional EQA program to ensure the accuracy of the DBS analysis that is not relevant to newborns screening. Consequently, for most DBS analyzes, there is no expert evaluation of laboratory characteristics.

\section{Conclusion}

A modern successful metabolomics study requires the simplification of sample preparation to prepare crafted samples, which will provide high-quality data. For metabolomic profiling DBS analysis using direct mass spectrometry applications is now broadly applied. As a less invasive sampling method, DBS offers a simple collection protocol, and it is easy to store and transfer samples. Analysis of an individual's blood metabolome using DBS method provides a valuable approach for real-time health status monitoring that is critical in the era of precision 
medicine. However, there are still a lot of challenges envisaged in sample preparation. Standardization for sample preparation is crucial in metabolomics study, which allows us to increase the number of measured and annotated compounds. In addition, quality control in a protocol is necessary to allow cross-laboratory comparisons.

\section{Acknowledgement}

The work was funded by the Program for Basic Research of State Academies of Sciences for 2013-2020.

\section{References}

1. Schnackenberg LK (2007) Global metabolic profiling and its role in systems biology to advance personalized medicine in the 21st century. Expert Rev Mol Diagn 7: 247-259.

2. Dunn WB, Broadhurst DI, Atherton HJ, Goodacre R, Griffin JL (2011) Systems level studies of mammalian metabolomes: the roles of mass spectrometry and nuclear magnetic resonance spectroscopy. Chem Soc Rev 40: 387-426.

3. Barrett D (2012) Advances in metabolic profiling. Bioanalysis 4: 643-644.

4. Schnackenberg LK, Beger RD (2006) Monitoring the health to disease continuum with global metabolic profiling and systems biology. Pharmacogenomics 7: 1077-1086.

5. Lokhov PG, Lisitsa AV, Archakov AI (2017) [Metabolomic blood test: purpose, implementation and interpretation of data]. Biomed Khim 63: 232-240. [Crossref]

6. Prakash AS, Pereira TN, Reilly PE, Seawright AA (1999) Pyrrolizidine alkaloids in human diet. Mutat Res 443: 53-67. [Crossref]

7. Borchers A, Teuber SS, Keen CL, Gershwin ME (2010) Food safety. Clin Rev Allergy Immunol 39: 95-141. [Crossref]

8. Ferguson LR, Philpott M (2008) Nutrition and mutagenesis. Annu Rev Nutr 28: 313329. [Crossref]

9. Crinnion MJ (2000) Environmental medicine, Part 2, Health effects of and protection from ubiquitous airborne solvent exposure. Altern Med Rev 5: 133-143.

10. Nielsen GD, Larsen ST, Olsen O, Løvik M, Poulsen LK, et al. (2007) Do indoor chemicals promote development of airway allergy? Indoor Air 17: 236-255.

11. Wallace LA, Pellizzari ED, Hartwell TD, Sparacino CM, Sheldon LS, et al. (1967) Personal exposure, indoor-outdoor relation- ships, and breath levels of toxic air pollutants measured for 355 persons in New Jersey. Atmospheric Environment 19: $1651-1661$.

12. Hill RH, Ashley DL, Head SL, Needham LL, Pirkle JL (1995) Dichlorobenzene exposure among 1,000 adults in the United States. Arch Environ Health 50: 277-280.

13. EPA (2012) Broad scan analysis of the FY82 national human adipose tissue survey specimens. EPA Office of Toxic Substances.

14. Ruhl RA, Chang CC, Halpern GM, Gershwin ME (1993) The sick building syndrome. II. Assessment and regulation of indoor air quality. J Asthma 30: 297-308.

15. Duehring C (1993) EPA stalls and industry hedges while consumers remain at risk, Informed Consent 1: 6-32.

16. Whitemore RW, Immerman FW, Camann DE, Bond AE, Lewis RG, et al. (1994) Non-occupational exposures to pesticides for residents of two U.S. cites. Arch Environ Contam Toxicol 26: 47-59.

17. Lokhov PG, Maslov DL, Trifonova OP, Balashova EE, Archakov AI (2014) Mass spectrometry analysis of blood low-molecular fraction as a method for unification of therapeutic drug monitoring. Biochemistry (Moscow) Supplement Series B: Biomedical Chemistry 8: 1-10

18. Trifonova OP, Lokhov PG, Archakov AI (2013) Metabolic profiling of human blood. Biochemistry (Moscow) Supplement Series B: Biomedical Chemistry 7: 179-186.

19. Trifonova O, Lokhov P, Archakov A (2013) Postgenomics diagnostics: metabolomics approaches to human blood profiling. Omics 17: 550-559.

20. Dunn WB, Broadhurst DI, Atherton HJ, Goodacre R, Grifn JL (2011) Systems level studies of mammalian metabolomes: The roles of mass spectrometry and nuclear magnetic resonance spectroscopy. Chem Soc Rev 40: 387-426.

21. Psychogios N, Hau DD, Peng J, Guo AC, Mandal R, et al. (2011) The human serum metabolome. PLoS One 6: e16957. [Crossref]

22. Lin L, Yu Q, Yan X, Hang W, Zheng J, et al. (2010) Direct infusion mass spectrometry or liquid chromatography mass spectrometry for human metabonomics? A serum metabonomic study of kidney cancer. Analyst 135: 2970-2978.
23. Dettmer K, Aronov PA, Hammock BD (2007) Mass spectrometry-based metabolomics. Mass Spectrom Rev 26: 51-78

24. Lokhov PG, Archakov AI (2008) [Mass spectrometry methods in metabolomics] Biomed Khim 54: 497-511. [Crossref]

25. Bowling FG, Thomas M (2014) Analyzing the metabolome. Methods Mol Biol 1168 31-45. [Crossref]

26. Nicholson JK, Lindon JC (2008) Systems biology: Metabonomics. Nature 455: 1054 1056. [Crossref]

27. Aretz I, Meierhofer D (2016) Advantages and pitfalls of mass spectrometry-based metabolome profiling in systems biology. Int J Mol Sci 17: E632.

28. Rhee EP (2015) Metabolomics and renal disease. Curr Opin Nephrol Hypertens 24: 371-379. [Crossref]

29. Zou HM, Zhang B, Xu XC, Sun YN, Xue S, et al. (2015) Urinary metabolomic strategy to evaluate Compound Danshen Dripping Pills for myocardial ischaemia in rats. $J$ Pharm Biomed Anal 112: 98-105.

30. Garcia-Canas V, Simo C, Castro-Puyana M, Cifuentes A (2014) Recent advances in the application of capillary electromigration methods for food analysis and Foodomics. Electrophoresis 35: 147-169.

31. Freemark M (2015) Metabolomics in nutrition research: biomarkers predicting mortality in children with severe acute malnutrition. Food Nutr Bull 36: 88-92.

32. Dunn WB, Broadhurst D, Begley P, Zelena E, Francis-McIntyre S, et al. (2011) Human serum metabolome. Procedures for large-scale metabolic profiling of serum and plasma using gas chromatography and liquid chromatography coupled to mass spectrometry. Nat Protoc 6: 1060-1083.

33. Guthrie KJR (1997) The PKU story: A crusade against mental retardation, Pasadena, USA.

34. Guthrie R, Susi A (1963) A simple phenylalanine method for detecting phenylketonuria in large populations of newborn infants. Pediatrics 32: 338-343.

35. Grüner N, Stambouli O, Ross RS (2015) Dried blood spots--preparing and processing for use in immunoassays and in molecular techniques. J Vis Exp. [Crossref]

36. Zhang T, Watson DG (2015) A short review of applications of liquid chromatography mass spectrometry based metabolomics techniques to the analysis of human urine. Analyst 140: 2907-2915.

37. Johnson SR, Lange BM (2015) Open-access metabolomics databases for natural product research: present capabilities and future potential. Front Bioeng Biotechnol 3: 22.

38. Mirnaghi FS, Caudy AA (2014) Challenges of analyzing different classes of metabolites by a single analytical method. Bioanalysis $6: 3393-3416$.

39. Han M, Jun SH, Song SH, Park HD, Park KU, et al. Ultraperformance liquid chromatography/tandem mass spectrometry for determination of sulfatides in dried blood spots from patients with metachromatic leukodystrophy, Rapid Commun. Mass Spectrom 28: 587-594.

40. Manicke NE, Abu-Rabie P, Spooner N, Ouyang Z, Cooks RG (2011) Quantitative analysis of therapeutic drugs in dried blood spot samples by paper spray mass spectrometry: an avenue to therapeutic drug monitoring. J Am Soc Mass Spectrom 22: $1501-1507$

41. Li Q, Cao D, Huang Y, Xu H, Yu C, et al. (2013) Development and validation of a sensitive LC-MS/MS method for determination of tacrolimus on dried blood spots. Biomed Chromatogr 27: 327-334. [Crossref]

42. Antunes MV, Raymundo S, de Oliveira V, Staudt DE, Gossling G, et al. (2015) Ultra-high performance liquid chromatography tandem mass spectrometric method for the determination of tamoxifen, N-desmethyltamoxifen, 4-hydroxytamoxifen and endoxifen in dried blood spots--development, validation and clinical application during breast cancer adjuvant therapy. Talanta 132: 775-784.

43. Sweetman L (2001) Newborn screening by tandem mass spectrometry: gaining experience. Clin Chem 47: 1937-1938. [Crossref]

44. Wagner M, Tonoli D, Varesio E, Hopfgartner G (2016) The use of mass spectrometry to analyze dried blood spots. Mass Spectrom Rev 35: 361-438. [Crossref]

45. Nishio H, Kodama S, Yokoyama S, Matsuo T, Mio T, et al. (1986) A simple method to diagnose adrenoleukodystrophy using a dried blood spot on filter paper. Clinica Chimica Acta 159: 77-82.

46. Millington DS, Kodo N, Norwood DL, Roe CR (1990) Tandem mass spectrometry: a new method for acylcarnitine profiling with potential for neonatal screening for inborn errors of metabolism. Journal of inherited metabolic disease 13: 321-324. 
47. Sosnoff CS, Ann Q, Bernert Jr JT, Powell MK, Miller BB, et al. (1996) Analysis of benzoylecgonine in dried blood spots by liquid chromatography-atmospheric pressure chemical ionization tandem mass spectrometry. Journal of analytical toxicology 20: 179-184.

48. Burnett JE (2011) Dried blood spot sampling: practical considerations and recommendation for use with preclinical studies. Bioanalysis 3: 1099-1107.

49. Zakaria R, Allen KJ, Koplin JJ, Roche1 P, Greaves RF (2016) Advantages and challenges of dried blood spot analysis by mass spectrometry across the total testing process. EJIFCC 27: 288-317.

50. Williams SR, McDade TW (2009) The use of dried blood spot sampling in the national social life, health, and aging project. J Gerontol B Psychol Sci Soc Sci 64: 131-136.

51. McDade TW, Williams S, Snodgrass JJ (2007) What a drop can do: dried blood spots as a minimally invasive method for integrating biomarkers into population-based research. Demography 44: 899-925.

52. Peng SH, Segura J, Farre M, de la Torre X (2000) Oral testosterone administration detected by testosterone glucuronidation measured in blood spots dried on filter paper. Clinical chemistry 46: 515-522.

53. Tretzel L, Thomas A, Geyer H, Gmeiner G, Forsdahl G, et al. (2014) Thevis, Use of dried blood spots in doping control analysis of anabolic steroid esters. J Pharm Biomed Anal 96: 21-30.

54. Cox HD, Rampton J, Eichner D (2013) Quantification of insulin-like growth factor-1 in dried blood spots for detection of growth hormone abuse in sport. Anal Bioanal Chem 405: 1949-1958.

55. Castro-Puyana M, Herrero M (2013) Metabolomics approaches based on mass spectrometry for food safety, quality and traceability. TrAC Trends in Analytical Chemistry 52: 74-87.

56. Fernández-Peralbo M, de Castro ML, Preparation of urine samples prior to targeted for untargeted metabolomics mass-spectrometry analysis. TrAC Trends in Analytical Chemistry 41: 75-85,

57. Deda O, Gika HG, Wilson ID, Theodoridis GA (2015) An overview of fecal sample preparation for global metabolic profiling. J Pharm Biomed Anal 10: 137-150.

58. NSQHS (2018) Safety and Quality Improvement Guide Patient Identification and Procedure Matching, Darlinghurst, NSW: Australian Commission on Safety and Quality in Health Care p. 28.

59. WHO (2010) Guidelines on Drawing Blood: Best Practices in Phlebotomy, Capillary sampling, World Health Organization.

60. Hannon WH (2013) NBS01-A6: Blood Collection on Filter Paper for Newborn Screening Programs, Approved Standard-Sixth Edition [Approved Guideline], Clinical and Laboratory Standards Institute.

61. CDC (1993) Laboratory Quality Assurance and Standardization Programs. Guidelines for the Shipment of Dried Blood Spot Specimens, USA: Centers for Disease Control and Prevention p. 4.

62. UNICEF (2009) Taking blood from infants for the HIV DNA PCR test - Standard Operating Procedures, National Health Laboratory Services, UNICEF, De Beers Fund p. 25.

63. WHO (2005) HIV rapid test training package: participant manual Blood Collection and handling - DBS, World Health Organisation p. 13.

64. Lehmann S, Delaby C, Vialaret J, Ducos J, Hirtz C (2013) Current and future use of "dried blood spot" analyses in clinical chemistry. Clin Chem Lab Med 51: 1897-1909.

65. Luckwell J, Danielsson A, Johnson B, Clegg S, Green M, et al. (2014) Applications and chemistry of cellulose papers for dried blood spots, in: Dried Blood Spots. John Wiley \& Sons, Inc pp: 332-343.

66. Mei JV, Zobel SD, Hall EM, De Jesus VR, Adam BW, et al. (2010) Performance properties of filter paper devices for whole blood collection. Bioanalysis 2: 1397-1403.

67. Ren X, Paehler T, Zimmer M, Guo Z, Zane P, et al. (2010) Impact of various factors on radioactivity distribution in different DBS papers. Bioanalysis 2: 1469-1475.

68. Vu DH, Koster RA, Alffenaar JW, Brouwers JR, Uges DR (2011) Determination of moxifloxacin in dried blood spots using LC-MS/MS and the impact of the hematocrit and blood volume. J Chromatogr B Analyt Technol Biomed Life Sci 879: 1063-1070.

69. Peng M, Liu L, Peng L (2012) Evaluation of factors influencing accuracy in the analysis of succinylacetone in dried blood spots. Clin Chim Acta 413: 1265-1269. [Crossref]

70. Holub M, Tuschl K, Ratschmann R, Strnadova KA, Muhl A, et al. (2006) Influence of hematocrit and localisation of punch in dried blood spots on levels of amino acids and acylcarnitines measured by tandem mass spectrometry. Clinica chimica acta 373: 27-31.
71. Parker SP, Cubitt WD (1999) The use of the dried blood spot sample in epidemiological studies. J Clin Pathol 52: 633-639.

72. Resnick L, Veren K, Salahuddin SZ, Tondreau S, Markham PD (1986) Stability and inactivation of HTLV-III/ LAV under clinical and laboratory environments. Jama 255: 1887-1891.

73. Garcia Boy R, Henseler J, Mattern R, Skopp G (2008) Determination of morphine and 6-acetylmorphine in blood with use of dried blood spots. Ther Drug Monit 30: 733-739. [Crossref]

74. Wong P, Pham R, Whitely C, Soto M, Salyers K, et al. Bruenner, (2011) Application of automated serial blood sampling and dried blood spot technique with liquid chromatography-tandem mass spectrometry for pharmacokinetic studies in mice. Journal of pharmaceutical and biomedical analysis 56: 604-608,

75. Heath AK, Williamson EJ, Ebeling PR, Kvaskoff D, Eyles DW, et al. (2014) Measurements of 25-hydroxyvitamin D concentrations in archived dried blood spots are reliable and accurately reflect those in plasma. The Journal of clinical endocrinology and metabolism 99: 3319-3324.

76. Prentice P, Turner C, Wong MC, Dalton RN (2013) Stability of metabolites in dried blood spots stored at different temperatures over a 2-year period. Bioanalysis 5: 1507-1514.

77. Michopoulos F, Theodoridis G, Smith CJ, Wilson ID (2011) Metabolite profiles from dried blood spots for metabonomic studies using UPLC combined with orthogonal acceleration ToF-MS: effects of different papers and sample storage stability Bioanalysis 3: 2757-2767.

78. Hollegaard MV, Grauholm J, Borglum A, Nyegaard M, Norgaard-Pedersen B, et al (2009) Genome-wide scans using archived neonatal dried blood spot samples. BMC genomics 10: 297.

79. D’Arienzo CJ, Ji QC, Discenza L, Cornelius G, Hynes J, et al. (2010) DBS sampling can be used to stabilize prodrugs in drug discovery rodent studies without the addition of esterase inhibitors. Bioanalysis 2: 1415-1422.

80. Liu G, Ji QC, Jemal M, Tymiak AA, Arnold ME (2011) Approach to evaluating dried blood spot sample stability during drying process and discovery of a treated card to maintain analyte stability by rapid on-card $\mathrm{pH}$ modification. Anal Chem 83: 9033-9038.

81. Elbin CS, Olivova P, Marashio CA, Cooper SK, Cullen E, et al. (2011) The effect of preparation, storage and shipping of dried blood spots on the activity of five lysosomal enzymes. Clinica Chimica Acta 412: 1207-1212.

82. O’Mara M, Hudson-Curtis B, Olson K, Yueh Y, Dunn J, et al. (2011) The effect of hematocrit and punch location on assay bias during quantitative bioanalysis of dried blood spot samples. Bioanalysis 3: 2335-2347.

83. Youhnovski N, Bergeron A, Furtado M, Garofolo F (2011) Precut dried blood spot (PCDBS): an alternative to dried blood spot (DBS) technique to overcome hematocrit impact. Rapid Commun. Mass Spectrom 25: 2951-2958.

84. Li F, Zulkoski J, Fast D, Michael S (2011) Perforated dried blood spots: a novel forma for accurate microsampling. Bioanalysis 3: 2321-2333.

85. Li F, Ploch S, Fast D, Michael S (2012) Perforated dried blood spot accurate microsampling: the concept and its applications in toxicokinetic sample collection. Journal of mass spectrometry 47: 655-667.

86. Zheng L, Yuan QC, Ji H, Mangus Y, Song C, et al. (2015) Center punch" and "whole spot" bioanalysis of apixaban in human dried blood spot samples by UHPLC-MS/ MS J Chromatogr B Analyt Technol Biomed Life Sci 988: 66-74.

87. Li, Henion J, Abbott R, Wang P (2012) The use of a membrane filtration device to form dried plasma spots for the quantitative determination of guanfacine in whole blood, Rapid Commun. Mass Spectrom 26: 1208-1212.

88. Shimadzu S (2016) Noviplex Cards: Shimadzu Oceania

89. Odoardi S, Anzillotti L, Strano-Rossi S (2014) Simplifying sample pretreatment application of dried blood spot (DBS) method to blood samples, including postmortem, for UHPLC-MS/MS analysis of drugs of abuse. Forensic Sci Int 243: 61-67.

90. Nirogi R, Kandikere V, Komarneni P, Aleti R, Padala N, et al. (2012) Exploring dried blood spot sampling technique for simultaneous quantification of antiretrovirals lamivudine, stavudine and nevirapine in a rodent pharmacokinetic study. Biomed. Chromatogr 26: 1472-1481

91. G la Marca, Giocaliere E, Villanelli F, Malvagia S, Funghini S, et al. D. (2012) Development of an UPLC-MS/ MS method for the determination of antibiotic ertapenem on dried blood spots. J Pharm Biomed Anal 61: 108-113.

92. Koseki N, Nakashima A, Nagae Y, Masuda N (2006) Simultaneous quantitative determination of cyclosporine A and its three main metabolites (AM1, AM4N and AM9) in human blood by liquid chromatography/mass spectrometry using a rapid sample processing method. Rapid Commun Mass Spectrom 20: 733-740. 
93. Li Y, Henion J, Abbott R, Wang P (2012) Semi-automated direct elution of dried blood spots for the quantitative determination of guanfacine in human blood. Bioanalysis 4: $1445-1456$.

94. Deglon J, Thomas A, Daali Y, Lauer E, Samer C, et al. (2011) Automated system for on-line desorption of dried blood spots applied to LC/MS/MS pharmacokinetic study of flurbiprofen and its metabolite. J Pharm Biomed Anal 54: 359-367.

95. J.Ht. Miller, Poston PA, Karnes HT (2011) Direct analysis of dried blood spots by inline desorption combined with high-resolution chromatography and mass spectrometry for quantification of maple syrup urine disease biomarkers leucine and isoleucine. Anal Bioanal Chem 400: 237-244.

96. Clark GT, Haynes JJ (2011) Utilization of DBS within drug discovery: a simple 2D-LC-MS/MS system to minimize blood- and paper-based matrix effects from FTA eluteâ,„£ DBS. Bioanalysis 3: 1253-1270.

97. Shokati T, Bodenberger N, Gadpaille H, Schniedewind B, Vinks AA, et al. (2015) Quantification of the immunosuppressant tacrolimus on dried blood Spots using LCMS/ MS. J Vis Exp 105: e52424.

98. Van Berkel GJ, Kertesz V, King RC (2009) High-throughput mode liquid microjunction surface sampling probe. Analytical Chemistry 81: 7096-7101.

99. Van Berkel GJ, Kertesz V (2013) Continuous-flow liquid microjunction surface sampling probe connected on-line with high-performance liquid chromatography/mass spectrometry for spatially resolved analysis of small molecules and proteins, Rapid Commun. Mass Spectrom 27: 1329-1334.

100. Edwards RL, Creese AJ, Baumert M, Griffiths P, Bunch J, et al. (2011) Hemoglobin variant analysis via direct surface sampling of dried blood spots coupled with highresolution mass spectrometry. Analytical Chemistry 83: 2265-2270.

101. Gaissmaier T, Siebenhaar M, Todorova V, Hullen V, Hopf C (2016) Therapeutic drug monitoring in dried blood spots using liquid microjunction surface sampling and high resolution mass spectrometry. Analyst 141: 892-901.

102. Kertesz V, Van Berkel GJ (2010) Fully automated liquid extraction-based surface sampling and ionization using a chip-based robotic nanoelectrospray platform. JMS 45: 252-260.

103. Ingels AS, Lambert WE, Stove CP (2010) Determination of gamma-hydroxybutyric acid in dried blood spots using a simple GC-MS method with direct "on spot" derivatization. Anal Bioanal Chem 398: 2173-2182. [Crossref]

104. Mess JN, Taillon MP, Côté C, Garofolo F (2012) Dried blood spot on-card derivatization: an alternative form of sample handling to overcome the instability of thiorphan in biological matrix. Biomed Chromatogr 26: 1617-1624. [Crossref]

105. Rhoden L, Antunes MV, Hidalgo P, Álvares da Silva C, Linden R (2014) Simple procedure for determination of valproic acid in dried blood spots by gas chromatography-mass spectrometry. J Pharm Biomed Anal 96: 207-212. [Crossref]

106. Déglon J, Lauer E, Thomas A, Mangin P, Staub C (2010) Use of the dried blood spo sampling process coupled with fast gas chromatography and negative-ion chemical ionization tandem mass spectrometry: application to fluoxetine, norfluoxetine, reboxetine, and paroxetine analysis. Anal Bioanal Chem 396: 2523-2532. [Crossref]

107. Kong ST, Lin HS, Ching J, Ho PC (2011) Evaluation of dried blood spots as sample matrix for gas chromatography/ mass spectrometry based metabolomic profiling. Anal Chem 83: 4314-4318. [Crossref]

108. Pitt JJ (2009) Principles and applications of liquid chromatography-mass spectrometry in clinical biochemistry. Clin Biochem Rev 30: 19-34.

109. Miller JM (2009) Chromatography with mass spectral detection (GC/MS and LC/ MS) in: Chromatography 1: 309-329.

110. Xu RN, Fan L, Rieser MJ, El-Shourbagy TA (2007) Recent advances in highthroughput quantitative bioanalysis by LC-MS/MS. J Pharm Biomed Anal 44: 342355. [Crossref]

111. Meesters RJ, Hooff GP (2013) State-of-the-art dried blood spot analysis: an overview of recent advances and future trends. Bioanalysis 5: 2187-2208. [Crossref]

112. Shushan B (2010) A review of clinical diagnostic applications of liquid chromatography-tandem mass spectrometry. Mass Spectrom Rev 29: 930-944. [Crossref]

113. Li F, McMahon C, Li F, Zulkoski J (2011) LC-MS/MS sensitivity enhancement using 2D-SCX/RPLC and its application in the assessment of pharmacokinetics of clonidine in dried blood spots. Bioanalysis 3: 1577-1586. [Crossref]

114. Oliveira RV, Henion J, Wickremsinhe E (2014) Fully-automated approach for online dried blood spot extraction and bioanalysis by two-dimensional-liquid chromatography coupled with high-resolution quadrupole time-of-flight mass spectrometry. Anal Chem 86: 1246-1253. [Crossref]
115. Saint-Marcoux F, Sauvage FL, Marquet P (2007) Current role of LC-MS in therapeutic drug monitoring. Anal Bioanal Chem 388: 1327-1349. [Crossref]

116. Wu AH, Gerona R, Armenian P, French D, Petrie M, et al. (2012) Role of liquid chromatography-high-resolution mass spectrometry (LC-HR/MS) in clinical toxicology. Clin Toxicol (Phila) 50: 733-742. [Crossref]

117. Taylor PJ, Tai CH, Franklin ME, Pillans PI (2011) The current role of liquid chromatography-tandem mass spectrometry in therapeutic drug monitoring of immunosuppressant and antiretroviral drugs. Clin Biochem 44: 14-20. [Crossref]

118. Keevil BG (2011) The analysis of dried blood spot samples using liquid chromatography tandem mass spectrometry. Clin Biochem 44: 110-118. [Crossref]

119. Crawford E, Gordon J, Wu JT, Musselman B, Liu R, et al. (2011) Direct analysis in real time coupled with dried spot sampling for bioanalysis in a drug-discovery setting. Bioanalysis 3: 1217-1226. [Crossref]

120. Cody RB, Dane AJ (2006) Direct Analysis in Real-Time Ion Source, in: Encyclopedia of Analytical Chemistry. [Crossref]

121. Wang C, Zhu H, Cai Z, Song F, Liu Z, et al. (2013) Newborn screening of phenylketonuria using direct analysis in real time (DART) mass spectrometry. Anal Bioanal Chem 405: 3159-3164.

122. Wild BJ, Green BN, Cooper EK, Lalloz MR, Erten S, et al. (2001) Rapid identification of hemoglobin variants by electrospray ionization mass spectrometry. Blood Cells Mol Dis 27: 691-704.

123. Takáts Z, Wiseman JM, Gologan B, Cooks RG (2004) Mass spectrometry sampling under ambient conditions with desorption electrospray ionization. Science 306: 471473. [Crossref]

124. Wiseman JM, Kennedy JH (2014) Analysis of dried blood spots using DESI mass spectrometry. Methods Mol Biol 1198: 291-297. [Crossref]

125. Wiseman JM, Evans CA, Bowen CL, Kennedy JH (2010) Direct analysis of dried blood spots utilizing desorption electrospray ionization (DESI) mass spectrometry. Analyst 135: 720-725.

126. Ranc V, Havlícek V, Bednar P, Lemr K (2008) Nano-desorption electrospray and kinetic method in chiral analysis of drugs in whole human blood samples. Eur J Mass Spectrom (Chichester) 14: 411-417. [Crossref]

127. Dénes J, Katona M, Hosszú A, Czuczy N, Takáts Z (2009) Analysis of biological fluids by direct combination of solid phase extraction and desorption electrospray ionization mass spectrometry. Anal Chem 81: 1669-1675. [Crossref]

128. Beecher CWW (2003) Metabolic profiling: Its role in biomarker discovery and gene function analysis. Springer, New York pp. 311-319.

129. Timmerman P, White S, Globig S, Lüdtke S, Brunet L, et al. (2011) EBF recommendation on the validation of bioanalytical methods for dried blood spots. Bioanalysis 3: 1567-1575. [Crossrref]

130. Abbott R, Smeraglia J, White S, Luedtke S, Brunet L, et al. (2010) Connecting strategies on dried blood spots. Bioanalysis 2: 1809-1816. [Crossref]

131. Timmerman P, White S, Cobb Z, Woods K, de Vries R, et al. (2014) European Bioanalysis Forum continued plans to support liquid microsampling. Bioanalysis 6: 1897-1900. [Crossref]

132. Abu-Rabie P, Denniff P, Spooner N, Brynjolffssen J, Galluzzo P, et al. (2011) Method of applying internal standard to dried matrix spot samples for use in quantitative bioanalysis. Anal Chem 83: 8779-8786. [Crossref]

133. Meesters R, Hooff G, van Huizen N, Gruters R, Luider T (2011) Impact of internal standard addition on dried blood spot analysis in bioanalytical method development. Bioanalysis 3: 2357-2364. [Crossref]

134. Zimmer D, Hassler S, Betschart B, Sack S, Fankhauser C (2013) Internal standar application to dried blood spots by spraying: investigation of the internal standard distribution. Bioanalysis 5: 711-719. [Crossref]

135. Heinig K, Bucheli F, Hartenbach R, Gajate-Perez A (2010) Determination of mycophenolic acid and its phenyl glucuronide in human plasma, ultrafiltrate, blood, DBS and dried plasma spots. Bioanalysis 2: 1423-1435. [Crossref]

136. Li W, Tse FL (2010) Dried blood spot sampling in combination with LC-MS/MS for quantitative analysis of small molecules. Biomed Chromatogr 24: 49-65. [Crossref]

Copyright: (C2018 Balashova EE. This is an open-access article distributed under the terms of the Creative Commons Attribution License, which permits unrestricted use, distribution, and reproduction in any medium, provided the original author and source are credited. 